\title{
Les membres de la Société Française de Physique et la culture scientifique
}

\author{
Daniel Hennequin $^{(1)}$ (daniel.hennequin@univ-lille.fr) et Fernande Vedel ${ }^{(2)}$ \\ (1) Président de la commission Culture scientifique de la SFP \\ PhLAM, UMR8523, Université de Lille, Bât. P5, Cité Scientifique, 59655 Villeneuve d'Ascq Cedex \\ (2) Professeur émérite, PIIM, UMR7345, Université d'Aix-Marseille, 13397 Marseille Cedex 20
}

Cet article présente la synthèse des résultats d'une enquête de la SFP auprès de ses adhérents, sur la culture scientifique dans la société française. Elle fait suite à une tribune sur ce sujet, parue en février 2018 dans le Huffington Post.

\section{La publication des résultats de cette enquête a été plusieurs fois reportée, pour des raisons techniques et éditoriales. Cette publication est donc très tardive, mais ses résultats quant à la perception de l'importance et du rôle de la culture scientifique dans notre société et au sein de la SFP restent d'actualité.}

Peu après la journée Sciences et Médias consacrée à la désinformation scientifique [1] en janvier 2018, une tribune "La culture scientifique est à reconquérir ", écrite par Virginie Tournay, membre du conseil scientifique de l'OPECST - Office parlementaire des choix scientifiques et technologiques -, et signée par une soixantaine de personnalités scientifiques et acteurs de la culture scientifique, a été publiée sur le site du Huffington Post [2]. La SFP avait préféré ne pas s'associer aux autres soutiens, jugeant l'argumentaire trop simpliste. Cette tribune avait été proposée au Monde, à Libération, au Point, aux Échos qui, soit l'ont refusée, soit ont demandé sa révision.

Les réactions à cette tribune ont été nombreuses sur les réseaux sociaux et les blogs $[3,4]$. Nous avions en particulier remarqué celle de Sylvestre Huet [4]. Le bureau de la SFP a alors proposé à sa commission Culture scientifique de demander à l'ensemble des adhérents leur avis sur la tribune, sur la réaction de la SFP et sur ce que pourraient être ses actions [5]. Cet article propose une synthèse des réponses, commentées par la commission.
Le titre de la tribune de V. Tournay est complété par le sous-titre "La reconquête de la culture scientifique nécessite de réfléchir à des mécanismes suscitant le désir de culture, à des médiations conduisant à une rencontre vivante avec la science ". En effet, cette tribune affirme que "la connaissance sociale de la science s'éloigne des considérants scientifiques ", autrement dit que tout public non scientifique a tendance actuellement à refuser la science, sa méthode et ses applications. Mais ce plaidoyer se limite à énumérer les " progrès technologiques " touchant à la vie de tous les jours, tout en dénonçant sans subtilité un "précautionisme " populiste, freinant des avancées scientifiques majeures. La tribune note que les travaux de l'OPECST ont peu d'impact sur les parlementaires et propose de créer une structure de médiation, notamment numérique, qui assisterait les médias, les politiques, les associations, et créerait un désir de culture scientifique auprès des étudiants et du grand public.

La riposte "Polémique sur la culture scientifique " de Sylvestre Huet, publiée sur le blog du Monde, pointe que le problème est réel, mais mal traité par V. Tournay. En fait, ce sujet est même central pour la société : il est en relation forte avec des défis immédiats, qui doivent être relevés en opérant démocratiquement des choix. $\mathrm{S}$. Huet considère qu'il faut changer de paradigme : on ne peut pas imaginer, par exemple, que nous pourrons continuer sur les logiques agricoles et technologiques actuelles. Le "noble et gai savoir ", respecté par l'opinion publique, offre son lot de turpitudes humaines (Bhopal, Tchernobyl...), et absoudre les technologies de leurs risques au nom de leurs origines scientifiques n'est pas judicieux. Grâce à la culture scientifique, l'État devrait être l'arbitre de conflits entre intérêts sociétaux, alors que les moteurs du profit et du pouvoir n'hésitent pas à tromper le consommateur : c'est l'agnotologie de R. Proctor, " la construction sociale de l'ignorance", organisée par exemple par les cigarettiers. L'expertise scientifique, à travers des agences pourvues de pouvoir, peut aussi être dévoyée : la solution est une action publique continue et la disponibilité d'experts indépendants issus d'une recherche publique bien financée. Devant l'étendue de l'inculture scientifique citée dans la tribune de V. Tournay, S. Huet rappelle que de fausses idées peuvent aussi être répandues par des scientifiques eux-mêmes (comme récemment Luc Montagnier). Il conclut en dénonçant une vision au service des technologies, qui pourrait contrarier le combat vers un débat public de qualité en induisant une attitude de défiance systématique. 


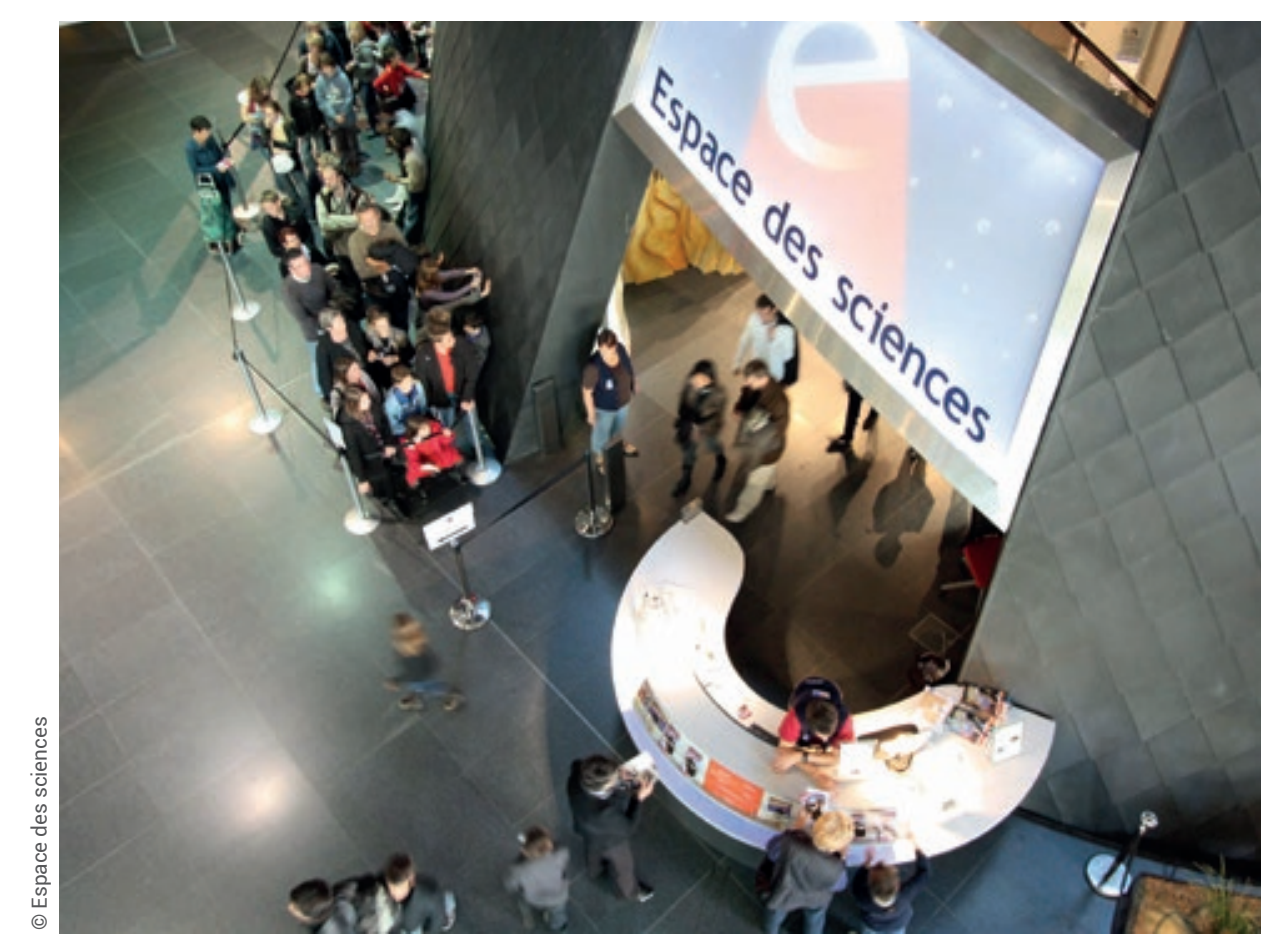

«Si la constitutionnalité [du principe de précaution] est désormais acquise, elle s'accompagne hélas d'un populisme précautionniste omniprésent...

Or, la précaution ne signifie pas résistance au changement, mais elle a pour visée la protection de la santé des personnes et de l'environnement. Ôter de façon systématique les vaccins, les produits pharmaceutiques, les micro-ondes, les fertilisants... à l'humanité aurait certainement des effets plus négatifs que positifs..." .

Virginie Tournay

\section{Ce que nous enseigne l'enquête}

Seulement 40 réponses sont parvenues à la SFP, soit un peu moins de 1,5\% des adhérents. $17 \%$ d'entre elles viennent de femmes (20,7\% des adhérents), 47\% de retraités (29\% des adhérents) et 7,5\% de doctorants ou de postdocs. Cette enquête ne reflète sans doute pas toutes les opinions des membres de la SFP. Malgré tout, les idées exprimées, parfois même par un seul individu, méritent réflexion.

En ce qui concerne la tribune de Virginie Tournay, une très large majorité des réponses (80\%, mais $100 \%$ des femmes) approuve les réserves de la SFP et sa position. La "brutalité " du texte, son " arrogance ", l'usage immodéré de l'argument d'autorité sont soulignés, ainsi qu'une confusion entre le titre et le contenu. On y parle assez peu de l'inculture scientifique, mais on $y$ mélange recherche scientifique, culture scientifique, applications technologiques ou encore usages des données de la science, ce qui produit un effet contre-productif dans la lutte contre les obscurantismes. La plupart des soutiens à la tribune partagent les mêmes analyses, mais prônent au contraire, compte tenu de la situation, une position radicale, comme celle de l'AFIS (Association française pour l'information scientifique), et estiment que la SFP devrait soutenir ce type de contribution qui dénonce l'obscurantisme, sans mettre en exergue des différences jugées minimes. Les recommandations proposées à la fin de la tribune (création d'une structure pour assister les médias, sensibilisation des étudiants, développement des médiations numériques) sont en général jugées assez timides, pas si drastiques que cela, voire dérisoires.

Les avis sur la réponse de Sylvestre Huet sont plutôt en accord avec ceux sur la tribune de Virginie Tournay : ceux qui émettent des réserves sur la tribune approuvent en général la réponse de Sylvestre Huet, et inversement. Cependant, la plupart considèrent que cette dernière ne constitue pas une véritable réponse. On $\mathrm{y}$ trouve des points assez justes, en particulier lorsqu'il s'agit de combattre le point de vue scientiste de la tribune et de relever qu'il faut parfois se poser des questions sur les retombées sociétales des avancées technologiques résultant de la recherche. Certains reprochent à S. Huet des positions contradictoires, tiraillées entre l'écologie et l'humanitaire. Les plus radicaux l'accusent d'argumentation fallacieuse, de sophisme par association.

Dans le détail, la tribune de Virginie Tournay constate a priori un recul de la culture scientifique dans l'ensemble de la société. De nombreuses réponses partagent cette affirmation. Les causes en seraient les médias, les politiques, l'absence d'intérêt de la population, l'existence de minorités opposées à la science et au progrès, les réseaux sociaux souvent utilisés par les activistes. Certains 
$\gg>$

évoquent le contexte, où l'on distingue culture scientifique et culture humaniste, et qui conduit à placer la première au second plan. On devrait pourtant accorder à la culture scientifique une place bien plus importante dans notre société technologique, appelée à prendre des décisions et faire des choix relevant de la science. Notons que le recul de la culture scientifique est loin d'être un constat indiscutable, et cette impression est peut-être bien une excellente illustration des biais de nos systèmes d'information contemporains, dénoncés par ces mêmes personnes qui « tombent dans le panneau ": en effet, des opinions qui s'expriment haut et fort peuvent donner cette impression, alors même qu'elles sont en réalité minoritaires. Pour évaluer l'image de la science dans le grand public, mieux vaut se tourner vers des études et des sondages. Citons par exemple le sondage "Regards et attentes des Français sur la recherche scientifique " [6], publié en octobre 2017, qui montre qu'une très grande majorité des Français a confiance dans les chercheurs et estime que la recherche scientifique est source d'espoir. L'absence d'intérêt de la population est plutôt contredite par les études réalisées. Une bonne référence dans le domaine est celle du Crédoc, d'avril 2013, intitulée « La curiosité scientifique des Français et leur désir de développer leurs connaissances " [7]. Il existe de nombreuses autres contributions : citons encore "Inculture scientifique des Français : pourquoi c'est un problème » [8].

Parmi les soutiens à la tribune de V. Tournay, il apparaît un fort sentiment critique vis-à-vis des médias. Le refus de publication de la tribune par Le Monde et Libération semble focaliser ce sentiment. Ce refus est alors attribué au fait que ces journaux ne publient que du sensationnel où l'émotion jouerait un rôle prépondérant, donnant ainsi parfois une visibilité trop importante à des avis très minoritaires. On reproche aux médias un manque de culture scientifique "abyssal ». Les journalistes scientifiques ne sont pas assez nombreux, les autres ont dans l'ensemble une formation littéraire. Ils sont parfois perçus comme étant de mauvaise foi. L'expertise scientifique est utilisée par les médias pour attiser des débats contradictoires en utilisant des arguments d'autorité, ce qui affaiblit la parole des scientifiques, alors que le fait scientifique est en deçà de la construction d'un avis et ne devrait pas entrer en compétition dans un débat contradictoire (et "démocratique ").
Enfin, les fausses informations scientifiques ne sont pas dénoncées. Il y a sans doute des journaux auxquels ces arguments s'appliquent, mais on peut difficilement reprocher au Monde de ne publier que des articles spectaculaires et d'ignorer les controverses. Rappelons que la SFP a cosigné plusieurs tribunes au cours des dernières années, dont celle sur l'avenir du Palais de la découverte [9], qui n'a rien de " sensationnel ". On peut aussi citer la tribune de Julien Bobroff [10], intitulée "Scientifiques et médiateurs doivent collaborer pour réinventer la vulgarisation ", publiée en juillet 2018, et qui elle aussi traite d'un problème de fond.

La commission Culture scientifique, et plus généralement la SFP, qui interagissent régulièrement avec des journalistes, ne peuvent pas approuver un reproche de manque de culture scientifique fait aux médias. La situation est en effet beaucoup plus subtile que cela, comme on pouvait s'en rendre compte en assistant aux quatre premières éditions des journées Sciences et Médias. Lors de la première édition, en 2012, nous avions par exemple découvert que c'était plutôt le rédacteur en chef de certains journaux qui posait problème, les journalistes se heurtant à un refus de publier leur article jugé "sans intérêt " [11]. À l'inverse, certains journaux font un travail tout à fait honnête, notamment en chassant et corrigeant les fausses informations (scientifiques ou pas). On peut citer les Décodeurs du Monde et l'initiative Cross Check, qui regroupe une vingtaine de médias français et internationaux. Les divergences qui apparaissent dans ces réponses relèvent davantage de la forme que du fond, mais ceux qui soutiennent la tribune de V. Tournay estiment que la forme doit l'emporter sur le fond quand la situation l'exige. Or, justement, lors de la journée Sciences et Médias consacrée à la désinformation scientifique [12], plusieurs intervenants ont mis en garde contre le manque d'efficacité d'une telle attitude. Le scientifique doit au contraire montrer l'exemple, en veillant à diffuser des informations étayées à 100\% par des arguments scientifiques, et en ne cédant par exemple jamais à l'argument d'autorité. Gary Dagorn, journaliste aux Décodeurs, s'interroge même sur la nécessité de toujours dénoncer la désinformation : est-ce que dans certains contextes, "décoder» des contrevérités ne produit pas l'effet inverse, à savoir celui de les amplifier [12] ?
« Cet appel à la culture scientifique peut donc facilement se caricaturer, à ses dépens, en une injonction brutale : acceptez tout ce que l'industrie et l'État vous proposent à base de technologies et de sciences... L'ennui, c'est que ce texte ne livre aucun exemple d'une précaution utile ou nécessaire... Cette asymétrie totale ne peut que susciter le trouble... »

Sylvestre Huet

\section{Les propositions des participants}

Si le « recul » de la culture scientifique en France est discutable, il n'en reste pas moins qu'elle est encore, en France, trop absente dans le public, dans les médias et chez les politiques, tout comme d'ailleurs le respect du savoir scientifique.

Dans les réponses à l'enquête, la plupart des solutions proposées pour combattre cette inculture concernent l'enseignement. Il faudrait donner plus de considération aux matières scientifiques, en augmentant les heures [de sciences] à tous les niveaux, et notamment dans les petites classes. Il faudrait done aussi améliorer la formation des enseignants, en particulier dans le primaire, où la plupart ont suivi une formation littéraire. Plus largement, un enseignement scientifique pourrait être donné dans les formations universitaires non scientifiques, comme en économie. La nouvelle réforme dans les programmes de lycées ne va pas dans le bon sens, puisqu'en réduisant les heures de sciences, elle va restreindre la culture scientifique des futurs scientifiques à une seule discipline !

L'accent est souvent mis sur l'apprentissage de l'esprit critique et de la démarche/méthode scientifique, et ce dès le primaire. On pourrait de ce point de vue s'inspirer de ce qu'on trouve ailleurs. Sur ce point, un témoignage 
mérite d'être livré intégralement : " [Je suis] actuellement aux USA, avec mon fils en CE1. Il a des cours de STEM (Science, Technology, Engineering and Maths). Ce sont des cours durant lesquels il va apprendre à coder, à construire (ponts, catapultes miniatures...). Des sorties dans des centres d'expériences avec un encadrement spécifique sont prévues (en particulier en chimie). Il a un emploi du temps et des enseignants spécifiques pour certaines matières, comme au collège. "En effet, des études montrent que lorsque les lacunes en science commencent très tôt dans la vie d'un enfant, elles persistent ensuite [13]. Il est donc important de faire découvrir la démarche scientifique dès le plus jeune âge. Une autre proposition concerne les sciences participatives, auxquelles on pourrait davantage faire appel pour familiariser le citoyen aux enjeux de la recherche scientifique. Rappelons que les sciences citoyennes, comme les appellent les Anglo-saxons, ont pour ambition de faire produire de la connaissance scientifique par tout citoyen qui le désire. Dans les domaines où de nombreux projets existent, comme en astronomie, en botanique ou en zoologie, les sciences participatives permettent incontestablement de sensibiliser les citoyens à la recherche scientifique et à ses méthodes. Malheureusement, en physique, les opportunités de faire avancer la recherche en impliquant le grand public sont rares. Mais il y a peut-être des pistes à explorer, comme celle du projet Foldit, en biochimie, qui permet de tester l'assemblage numérique de nouvelles protéines au travers d'un jeu vidéo public [14].

\section{Des pistes pour agir}

Lorsqu'il s'agit du rôle que pourrait jouer la SFP dans tous les domaines évoqués cidessus, les réponses sont unanimes : elle n'en fait pas assez.

La SFP devrait amplifier son rôle critique vis-à-vis des thèses obscurantistes que l'on trouve sur la toile. Elle pourrait en particulier être beaucoup plus active sur les réseaux sociaux, en dénonçant ces thèses, et plus généralement les conspirationnistes ou les pratiques prédatrices (éditeurs, conférences). Elle pourrait aussi maintenir un site de réfutation sur des questions de physique (Terre plate, mouvement perpétuel...). De ce point de vue, elle devrait aussi se rapprocher de l'AFIS.

Il faudrait lancer des débats sur les aspects sociaux, économiques, politiques, éthiques, culturels, sur l'introduction massive des nouvelles technologies dans la société. La SFP pourrait être " experte en expertise " et plus présente dans les débats de société où l'éducation interfêre avec la politique, et où l'information contenue dans les journaux est au mieux déficiente : les sources d'énergie, la reconstruction de l'industrie française, les questions de science et défense, les programmes de physique au collège et au lycée...

\section{Conclusion}

Le premier enseignement de cette enquête semble être que les membres de la SFP se soucient fort peu de culture scientifique. Cette remarque peut sûrement être tempérée par l'espoir que nombreux sont les adhérents qui se sentent concernés mais n'ont pas trouvé le temps de répondre à l'enquête. Et l'on touche bien là au principal frein à la mise en place des propositions ci-dessus : la SFP est une association qui, comme toutes les associations, ne peut compter que sur l'engagement et le soutien financier de ses membres. Pour atteindre ces objectifs, il lui faudrait davantage de personnes impliquées, et ça, c'est l'affaire de tous ! La commission Culture scientifique est ouverte à tous et à toutes : n'hésitez pas à nous rejoindre !

On peut cependant remarquer que dans les réponses à cette enquête, nos adhérents n'évoquent pas le manque d'engagement des scientifiques eux-mêmes, et en particulier des physiciens. Pourtant la diffusion de la culture scientifique est aussi de notre ressort, et l'on dispose dans ce domaine d'une très grande marge de progression. La commission Culture scientifique continuera à mener une réflexion sur les résultats de cette enquête, et engagera, sur les sujets qui la concernent et dans la mesure de ses moyens, des actions allant dans le sens des orientations suggérées.

\section{Références}

1 - www.sciencesetmedias.org

La session Culture scientifique du Congrès général de la SFP, à Nantes, en juillet 2019, était aussi consacrée à la désinformation scientifique.

2- www.huffingtonpost.fr/virginie-tournay/ ne-nous-reposons-pas-sur-nos-lauriersen-france-la-culture-scientifique-est-areconquerir_a_23369215/

3• https://blogs.mediapart.fr/michel-pinault/ blog/010318/crise-de-la-culturescientifique-crise-de-la-science

4- http://huet.blog.lemonde.fr/2018/02/27/ polemique-sur-la-culture-scientifique/

5. Lien vers le texte de la consultation https://www.sfpnet.fr/uploads/ tinymce/2020/sfp-culture-enquete.pdf
6. www.opinion-way.com/fr/sondage-dopinion/sondages-publies/opinionsociete/societe/opinionway-pourquattrocento-regards-et-attentes-desfrancais-sur-la-recherche-scientifiqueoctobre-2017.html

7• www.credoc.fr/pdf/Rapp/R289.pdf

8• www.contrepoints.org/2018/03/11/ 311455-inculture-scientifique-un-probleme

9• www.lemonde.fr/sciences/article/ 2018/06/19/la-mutation-du-palais-dela-decouverte-ne-doit-pas-alterer-sonadn_5317678_1650684.html

10 - www.lemonde.fr/sciences/article/ 2018/07/12/scientifiques-et-mediateursdoivent-collaborer-pour-reinventer-lavulgarisation_5330195_1650684.html
11 D. Bideau et al., « Une journée "Science et Médias" au Palais de la découverte", Reflets de la physique 31 (2012) 26-29. www.refletsdelaphysique.fr/articles/ refdp/pdf/2012/04/refdp201231p26.pdf

12• R. Farhi, « Comment lutter contre la désinformation scientifique? Journée Sciences et Médias 2018 ", Reflets de la physique, 58 (2018) 33-37. www.refletsdelaphysique.fr/articles/ refdp/pdf/2018/03/refdp201858p33.pdf

13. P. Morgan et al., "Science Achievement Gaps Begin Very Early, Persist, and Are Largely Explained by Modifiable Factors", Educational Researcher, 45 (2016) 18-35.

14・ https://fold.it/portal/ 